\title{
Hyaluronan-mediated motility: A target in oral squamous cell carcinoma
}

\author{
YUKIO YAMANO $^{1}$, KATSUHIRO UZAWA ${ }^{1,3}$, KEIJI SHINOZUKA ${ }^{1}$, KAZUAKI FUSHIMI ${ }^{1}$, \\ TAKASHI ISHIGAMI ${ }^{1}$, HITOMI NOMURA ${ }^{1}$, KATSUNORI OGAWARA ${ }^{1}$, MASASHI SHIIBA ${ }^{1}$, \\ HIDETAKA YOKOE ${ }^{3}$ and HIDEKI TANZAWA ${ }^{1-3}$ \\ ${ }^{1}$ Department of Clinical Molecular Biology, Graduate School of Medicine, ${ }^{2}$ Center of Excellence (COE) \\ Program in The 21st Century, Graduate School of Medicine, Chiba University; ${ }^{3}$ Division of Dentistry \\ and Oral-Maxillofacial Surgery, Chiba University Hospital, 1-8-1 Inohana, Chuo-ku, Chiba 260-8670, Japan
}

Received December 27, 2007; Accepted February 21, 2008

\begin{abstract}
To characterize cancer-related gene expression changes in oral squamous cell carcinomas (OSCCs), we compared the gene expression profiles in OSCC-derived cell lines with human normal oral keratinocytes (HNOKs). Microarray analysis identified 166 genes that were up-regulated in OSCC-derived cell lines. Gene ontology analysis showed that cancer-related function had the highest significance. Among the genes mapped to the cancer-related network with the highest significance, the receptor for hyaluronan-mediated motility (RHAMM) was evaluated further for mRNA and protein expression in the OSCC cell lines, primary OSCCs. Overexpression of RHAMM protein was observed in all cell lines compared to HNOKs. Immunohistochemical analysis showed highly expressed RHAMM in primary OSCCs, whereas most corresponding normal tissues had no or significant down-regulation of protein immunoreactivity. Real-time quantitative reverse transcriptase-polymerase chain reaction data agreed with the protein expression. Moreover, the RHAMM expression status was correlated with the TNM stage $(\mathrm{P}<0.001)$. The results suggested that RHAMM expression may be correlated with tumor aggressiveness and offer clues to the development of new treatments for human OSCCs.
\end{abstract}

\section{Introduction}

Head and neck cancers are the sixth most common cancers worldwide and major causes of morbidity and mortality (1).

Correspondence to: Dr Katsuhiro Uzawa, Chiba University, 1-8-1 Inohana, Chuo-ku, Chiba 260-8670, Japan

E-mail: uzawak@faculty.chiba-u.jp

Key words: oral squamous cell carcinoma, receptor for hyaluronan-mediated motility, pathway analysis, microarray analysis, qRT-PCR, Western blot, immunohistochemistry
Cancers of the oral cavity account for $40 \%$ of head and neck cancers including squamous cell carcinoma (2). Oral squamous cell carcinomas (OSCCs) have been identified as major global threats to public health, the prognosis of which have not improved in decades (3). With the currently available clinical assessment and treatment methods, a third of all cases of oral cancer are diagnosed only after becoming locally advanced $(4,5)$. In addition, aggressive treatment of OSCCs is controversial because it can lead to severe morbidity and disfigurement. As a result, many patients with OSCCs are either overtreated or untreated, with substantial personal and socioeconomic implications. A number of etiologic factors have been implicated in the development of OSCCs, such as the use of tobacco, alcohol, or the presence of incompatible prosthetic materials $(6,7)$. However, some patients develop OSCC without risk factors, suggesting that host susceptibility may play a role. Molecular alterations in a number of oncogenes and tumor suppressor genes associated with the development of OSCC could be important clues for addressing these problems $(7,8)$. Comprehensive and exhaustive expression studies of numerous genes, including functionally unknown genes, are essential to understand the complexity and polymorphisms of OSCC.

Microarray technologies have been used widely for comprehensive gene expression analysis that enables researchers to simultaneously analyze changes in thousands of genes and identify significant patterns. However, previous microarray studies performed in OSCCs have produced lists of differentially expressed genes (9-11) but failed to establish how these genes form regulatory networks. Moreover, these studies have ignored the genes that do not pass the randomly or empirically determined criteria for gene selection. We performed microarray analysis using high-density Affymetrix Human Genome-U133A plus 2.0 GeneChip arrays containing 54,675 probe sets (Affymetrix, Santa Clara, CA) to compare gene expression patterns among the OSCC-derived cell lines and human normal oral keratinocytes (HNOKs). We also adopted a computational tool, Ingenuity Pathway Analysis (IPA) software (Ingenuity Systems, Mountain View, CA) to identify regulatory networks of differentially expressed genes related to OSCCs. 
We analyzed the gene expression profiles of OSCCs using microarray technology to identify genes associated with oral carcinogenesis, which then were analyzed for network and gene ontology by IPA to identify networks of interacting genes and other functional groups. We also verified experimentally that the extracellular matrix-associated protein, receptor for hyaluronan-mediated motility (RHAMM), is OSCC up-regulated in humans and also plays an important role in tumor progression.

\section{Materials and methods}

Tissue specimens and cell lines. Tumors and patient-matched normal epithelium were obtained during surgical resection at Chiba University Hospital after patient informed consent was obtained under a protocol reviewed and approved by the institutional review board of Chiba University. The resected tissues were divided into two parts, one of which was frozen immediately after removal of the surrounding normal tissues and stored at $-80^{\circ} \mathrm{C}$ until protein isolation, and the second of which was fixed in $10 \%$ buffered formaldehyde solution for pathologic diagnosis and immunohistochemical staining. Histopathologic diagnosis of each neoplastic tissue was performed according to the World Health Organization criteria by the Department of Pathology, Chiba University Hospital. Clinicopathologic staging was determined by the TNM classification of the International Union against Cancer. All patients had SCC that was histologically confirmed, and tumor samples were checked to ensure that tumor tissue was present in $>80 \%$ of the specimens. The OSCC-derived cell lines used in this study were HSC-2, HSC-3, HSC-4, Ca9-22, (Human Science Research Resources Bank, Osaka, Japan), H1, and Sa3 (provided by Dr Fujita at Wakayama Medical University, Wakayama, Japan). All OSCC-derived cell lines were grown in Dulbecco's modified Eagle's medium/F-12 HAM (Sigma-Aldrich Co., St. Louis, MO) supplemented with $10 \%$ fetal bovine serum (Sigma) and 50 units $/ \mathrm{ml}$ penicillin and streptomycin (Sigma). Healthy oral gingival specimens were collected from 22- to 35-year-old patients at Chiba University Hospital. The institutional review board of Chiba University approved all relevant protocols. Five independent HNOKs were primary cultured and maintained in defined keratinocyte-SFM (Gibco-BRL, Gaithusberg, Germany) (12).

Affymetrix GeneChip hybridization. Double-stranded cDNA was synthesized from $20 \mu \mathrm{g}$ of total RNA using the Superscript Choice system (Invitrogen Life Technologies, Carlsbad, CA, USA). After phenol/chloroform extraction and ethanol precipitation, a biotin-labeled in vitro transcription reaction was carried out using the cDNA template (Enzo Bioarray, Farmingdale, NY). cRNA ( $7 \mu \mathrm{g})$ was fragmented according to Affymetrix protocols and added to the recommended hybridization mixture. Expression profiles were created using the Human Genome U 133 Plus 2.0 arrays containing 54,675 probe sets (Affymetrix). Arrays were stained with phycoerythrin-streptavidin, and the signal intensity was amplified by treatment with a biotin-conjugated anti-streptavidin antibody followed by a second staining with phycoerythrinstreptavidin. Arrays stained a second time were scanned using the Affymetrix GeneChip Scanner 3000.
Data analysis. GeneChip analysis was performed based on the Affymetrix GeneChip Manual with Microarray Analysis Suite 5.0, Data Mining Tool 2.0, and Microarray Database software. All genes on the GeneChip were globally normalized and scaled to a signal intensity of 500. The Microarray Analysis Suite software used Wilcoxon's test to generate detected (present or absent) calls and used the calls to statistically determine if a transcript was expressed or not. After being filtered through a 'present' call $(\mathrm{P}<0.05)$, the expression data were analyzed using GeneChip Operating Software 1.1 (Affymetrix) and GeneSpring 6.1 (Silicon Genetics, Redwood City, CA). Fold changes were calculated by comparing transcripts between OSCC-derived cell lines and HNOKs.

Network and gene ontology analysis. The list of up-regulated genes in OSCC identified by microarray analysis was used for network and gene ontology analysis. Gene accession numbers were imported into the IPA software. The genes were categorized based on location, cellular components, and reported or suggested biochemical, biologic, and molecular functions using the software. The identified genes also were mapped to genetic networks available in the IPA database and ranked by score. The score is the probability that a collection of genes equal to or greater than the number in a network can be achieved by chance alone. A score of 3 indicates that there is a $1 / 1000$ chance that the focus genes are in a network due to random chance. Therefore, scores of 3 or higher have a $99.9 \%$ confidence level of not being generated by random chance alone. This score was used as the cut-off for identifying gene networks.

Protein and $m R N A$ extraction. Protein was extracted when the cells reached $80-90 \%$ confluence; they were washed twice with phosphate-buffered saline (PBS), scraped into a tube and centrifuged briefly. The cell pellets were incubated for $30 \mathrm{~min}$ in a lysis buffer containing $7 \mathrm{M}$ urea, $2 \mathrm{M}$ thiourea, $4 \% \mathrm{w} / \mathrm{v}$ CHAPS, and $10 \mathrm{mM}$ Tris $\mathrm{pH} 8.0$, and lysed by sonication ( $3 \times 10 \mathrm{sec}$ pulses on ice). The sample was centrifuged at 13,000 rpm for $20 \mathrm{~min}$. The supernatant containing the cell proteins then was recovered and the protein concentration was measured with a Protein Assay Kit (Bio-Rad Laboratories, Hercules, CA) and adjusted to $1 \mathrm{mg} / \mathrm{ml}$ with lysis buffer. The $\mathrm{pH}$ of the protein sample was adjusted to 8.5 with $30 \mathrm{mM}$ Tris- $\mathrm{HCl}$. Total RNA was extracted using TRIzol Reagent (Invitrogen Life Technologies) according to the manufacturer's instructions. The extracted RNA samples were stored separately at $-80^{\circ} \mathrm{C}$ until use.

$m R N A$ expression analysis. Real-time quantitative reverse transcriptase-polymerase chain reaction (qRT-PCR) was performed to evaluate the expression level of RHAMM mRNA in the OSCC-derived cell lines (HSC-2, HSC-3, HSC-4, Ca9-22, H1 and Sa3) and HNOKs. In addition, primary tumors and paired specimens of normal oral tissues from 50 patients were also evaluated. qRT-PCR was performed with a single method using a LightCycler FastStart DNA Master SYBR-Green 1 Kit (Roche Diagnostics GmbH, Mannheim, Germany), according to the procedure provided by the manufacturer. The primer sequences used to analyze 
RHAMM mRNA expression were forward 5'-TTCTGA ACCCTTTGGCTGG-3' and reverse 5'-ACAAGCCAAG GTGTTTTAGCC-3'. The sequence of specific primers was checked before use to avoid amplification of genomic DNA or pseudogenes by Primer3 program (available at http://www-genome.wi.mit.edu/cgi-bin/primer/ primer3_www.cgi). Amplified products were analyzed by $3 \%$ agarose gel electrophoresis to ascertain size and purity. The PCR reactions using the LightCycler (Roche) apparatus were carried out in a final volume of $20 \mu 1$ of a reaction mixture consisting of $2 \mu \mathrm{l}$ of FirstStart DNA Master SYBR-Green I mix, $3 \mathrm{mM} \mathrm{MgCl}_{2}$ and $0.2 \mu \mathrm{l}$ of the primers, according to the manufacturer's instructions. The reaction mixture then was loaded into glass capillary tubes and subjected to an initial denaturation at $95^{\circ} \mathrm{C}$ for $10 \mathrm{~min}$, followed by 45 rounds of amplification at $95^{\circ} \mathrm{C}(10 \mathrm{sec})$ for denaturation, $63^{\circ} \mathrm{C}(10 \mathrm{sec})$ for annealing, and $72^{\circ} \mathrm{C}$ for extension, with a temperature slope of $20^{\circ} \mathrm{C} / \mathrm{sec}$, performed in the LightCycler. The transcript amount for the RHAMM gene was estimated from the respective standard curves and normalized to the glyceraldehyde-3-phosphate dehydrogenase (GAPDH) (forward 5'-CATCTCTGCCCCCTCTGCTGA-3' and reverse 5'-GGATGACCTTGCCCACAGCCT-3') transcript amount determined in corresponding samples. The significance of the gene expression levels between RHAMM-positive and negative cases was calculated with Mann-Whitney U test, with $\mathrm{P}<0.05$ considered significant.

Western blot analysis. Protein extracts were electrophoresed on $11 \%$ sodium dodecyl sulfate-polyacrylamide gel electrophoresis gels, transferred to PVDF membranes (Bio-Rad), and blocked for $1 \mathrm{~h}$ at room temperature in 5\% skim milk. Immunoblot PVDF membranes were washed with $0.1 \%$ Tween-20 in TBS (TBS-T) five times, and $2 \mu \mathrm{g} / \mathrm{ml}$ affinitypurified goat anti-RHAMM polyclonal antibody (Santa Cruz Biotechnology, Inc.) was added directly to the TBS-T solution for $2 \mathrm{~h}$ at room temperature. PVDF membranes were washed again and incubated with a 1:1000 of horseradish peroxidase-conjugated anti-goat IgG Envision+ (Dako Japan Inc., Kyoto, Japan) as a secondary antibody for $20 \mathrm{~min}$ at room temperature. Finally, the membranes were incubated with the ECL+ horseradish peroxidase substrate solution included in the ECL+ kit (Amersham Biosciences UK Ltd., UK), and immunoblotting was visualized by exposing the membranes to Hyperfilm (Amersham) $(13,14)$. Triplicate examinations were performed to confirm the specificity of the antibody.

Immunohistochemistry. Immunohistochemical staining was performed on 4- $\mu \mathrm{m}$ sections of paraffin-embedded specimens using goat anti-RHAMM polyclonal antibody. Briefly, after deparaffinization and hydration, the slides were treated with endogenous peroxidase in $0.3 \% \mathrm{H}_{2} \mathrm{O}_{2}$ for $30 \mathrm{~min}$, after which the sections were blocked for $2 \mathrm{~h}$ at room temperature with $1.5 \%$ blocking serum (Santa Cruz) in PBS before reacting with anti-RHAMM antibody (1:100 dilution) at room temperature in a moist chamber overnight. Upon incubation with the primary antibody, the specimens were washed three times in PBS and treated with Envision reagent followed by color development in 3,3'-diaminobenzidine tetrahydrochloride (Dako). Finally, the slides were lightly counterstained with hematoxylin, dehydrated with ethanol, cleaned with xylene and mounted. As a negative control, duplicate sections were immunostained without exposure to primary antibodies. To quantitate the state of RHAMM protein expression, the mean percentage of positive tumor cells was determined in at least five random fields at x400 magnification in each section. The intensity of the RHAMMimmunoreaction was scored as: $1+$, weak; $2+$, moderate; and $3+$, intense. The percentage of positive tumor cells and the staining intensity then were multiplied to produce a RHAMMimmunohistochemical staining score $(15,16)$. Cases with a RHAMM score $>89.12$ (the highest score for normal tissue) were defined as positive. These judgments were made by two independent pathologists, neither of whom had any knowledge or information pertaining to the patients' clinical status. Statistical significance was evaluated by Fisher's exact test, or Mann-Whitney $\mathrm{U}$ test. $\mathrm{P}<0.05$ was considered significant.

\section{Results}

Up-regulated gene expression in OSCC-derived cell lines. To identify differentially expressed genes between HNOKs and OSCC-derived cell lines, we performed global gene expression analysis using Affymetrix GeneChips containing $>54,000$ probe sets $(39,000$ transcripts and 33,000 annotated genes) with RNAs isolated from 4 OSCC-derived cell lines (HSC-2, HSC-3, H-1 and Sa-3). Control RNAs consisted of HNOK RNA extracted from healthy oral gingival specimens. Expression data were analyzed using the GeneChip Operating Software 1.1 and GeneSpring 6.1.

The results of microarray analysis showed that the expression levels of 166 genes were elevated $\geq 5$-fold in all OSCC derived cell lines compared with the HNOKs (data not shown).

Network and gene ontology analysis. Based on all genes identified as described previously (named focus genes), new and expanded pathway maps and connections and specific gene-gene interactions were inferred, functionally analyzed, and used to build on the existing pathway using the IPA knowledge base. To generate networks, the knowledge base was queried for interactions between the focus genes and all other gene objects stored in the base. Seven networks were significant in OSCC, in that they had more of the identified genes present than would be expected by chance (Table I). We also performed gene ontology analysis of 166 focus genes using the IPA tool. Thirty-six up-regulated genes were associated with cancer-related molecular function (Table II). The cancer-related function had the highest $\mathrm{P}$-value ( $\mathrm{P}=2.36 \mathrm{E}-06$ to $1.23 \mathrm{E}-02)$. Furthermore, to investigate the network of 36 cancer-related genes, we performed network analysis and identified one network (Fig. 1) that included 13 cancer-related genes (Table III). Among the genes mapped to the highest-significance cancer-related network, RHAMM was analyzed further.

$m R N A$ expression analysis. We examined the expression levels of RHAMM mRNA in six OSCC-derived cell lines, 
Table I. Seven networks formed by over-expressed genes in OSCC cell lines.

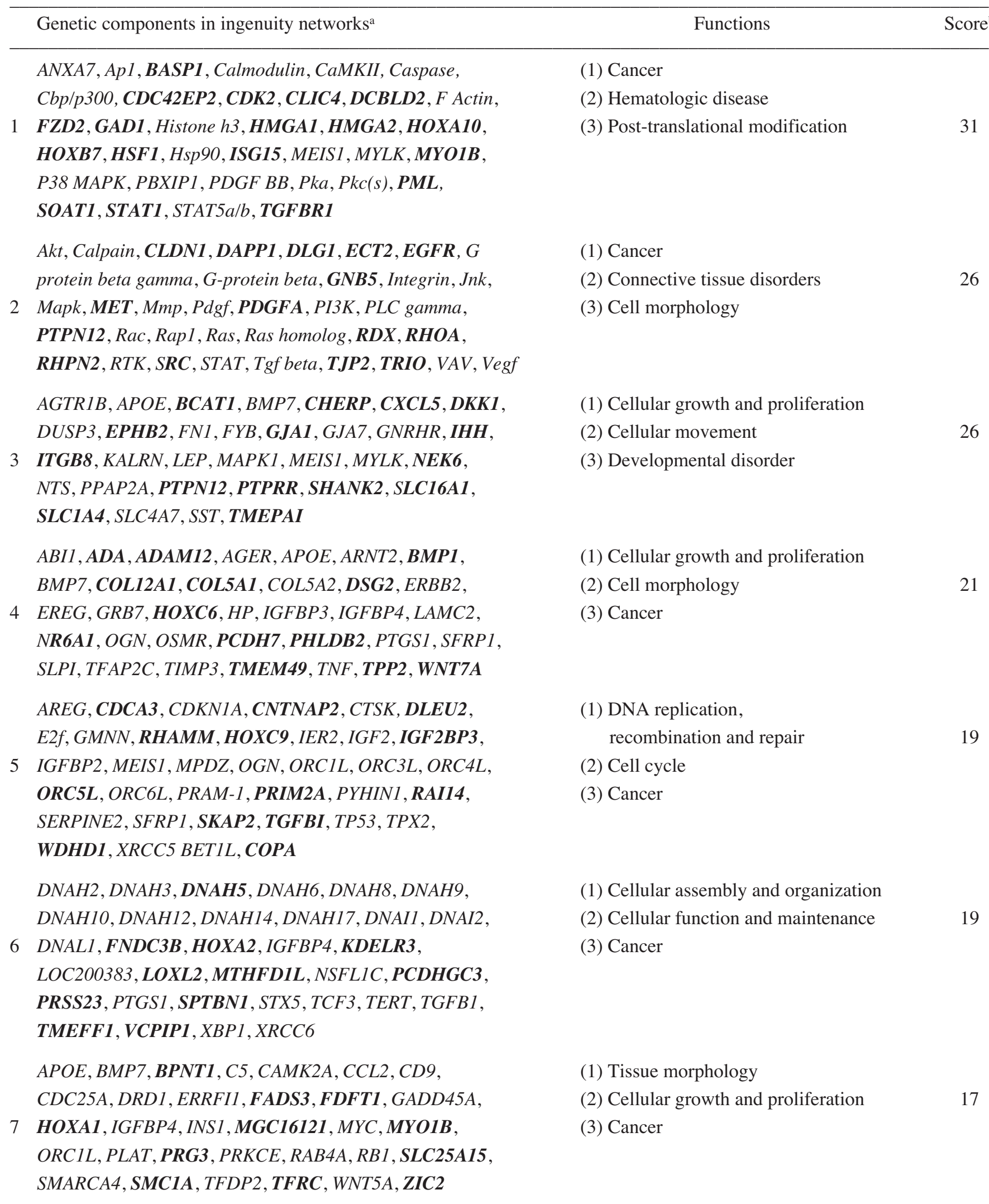

${ }^{\mathrm{a}}$ Genes in boldface were identified by microarray analysis to be expressed differentially $>5$-fold in OSCCs. Other genes were either not on the expression array or not significantly regulated. ${ }^{\mathrm{b}} \mathrm{A}$ score $>3$ was considered significant.

HNOKs, primary tumors, and paired normal oral tissues from 50 patients with OSCC using qRT-PCR analysis. Significant up-regulation of RHAMM expression was observed in all OSCC cell lines examined compared with the HNOKs used 
Table II. Five most frequently observed molecular functions in the identified genes related to disease and disorders.

\begin{tabular}{lcr}
\hline Molecular function & P-value & Gene \\
\hline 1. Cancer & 2.36E-06 to 1.23E-02 & 36 \\
2. Connective tissue disorders & $1.57 \mathrm{E}-05$ to 1.23E-03 & 11 \\
3. Hematologic disease & 3.79E-05 to 1.23E-02 & 9 \\
4. Gastrointestional disorders & 5.38E-05 to 1.23E-02 & 9 \\
5. Skeletal and muscular disorders & 1.13E-04 to 6.19E-03 & 3 \\
\hline
\end{tabular}

as a control (Fig. 2A). Data are expressed as the means \pm SD of two independent experiments with samples in triplicate. In addition, similar to the OSCC cell lines, the RHAMM expression levels were up-regulated in primary tumors compared with matched normal tissues $(\mathrm{P}<0.005$; MannWhitney U test) (Fig. 2B). qRT-PCR analysis revealed upregulation of RHAMM expression in $42(84 \%)$ of 50 primary OSCCs compared with matched normal tissues. The relative mRNA expression levels in the normal tissues and primary OSCCs ranged from 0.08 to 7.26 (median, 2.32) and 0.53 to 27.2 (median, 5.11), respectively. mRNA expression levels were normalized to GAPDH.

Analysis of protein expression of RHAMM in OSCC-derived cell lines. To investigate RHAMM protein expression status in the six OSCC-derived cell lines (HSC-2, HSC-3, HSC-4, Ca9-22, Sa-3 and H1) (n=6) and the HNOKs, we performed Western blot analysis. Fig. 3A shows representative results.

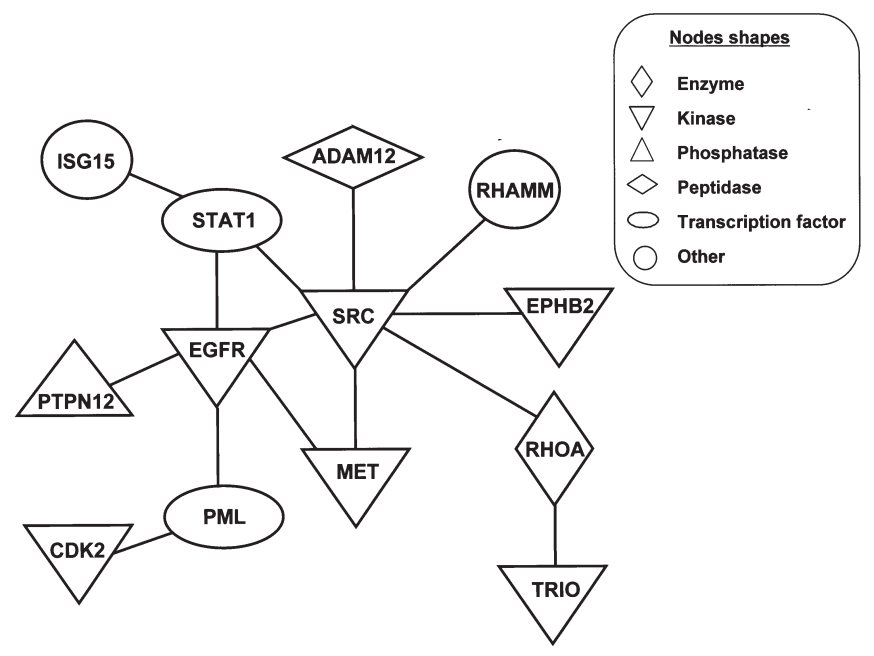

Figure 1. Genes up-regulated in OSCC-derived cell lines compared with HNOKs and related to cancer function of OSCC in gene ontology analysis. The functional relationship between gene products based on known interactions in IPA knowledge is described. Shapes of nodes symbolize functional class of gene product.

The size of the band was detected as $85-90 \mathrm{kDa}$, as reported previously (17). A significant increase in RHAMM expression was observed in all OSCC cell lines compared with the HNOKs.

Immunohistochemistry. A total of 52 patients with OSCC were identified for whom there was adequate histologic material available for immunohistochemical analysis with corresponding normal tissues. The correlation between the clinicopathologic characteristics of patients with OSCC and

Table III. Thirteen cancer-related genes.

\begin{tabular}{|c|c|c|c|}
\hline Affymetrix no. & Gene & Description & Fold change \\
\hline 211607_x_at & $E G F R$ & Epidermal growth factor receptor & 71.971 \\
\hline 211804_s_at & $C D K 2$ & Cyclin-dependent kinase 2 & 17.000 \\
\hline 207165_at & RHAMM & Hyaluronan-mediated motility receptor (RHAMM) & 13.166 \\
\hline 203510_at & $M E T$ & Met proto-oncogene & 13.159 \\
\hline 211165_x_at & EPHB2 & EPH receptor B2 & 13.011 \\
\hline 208178_x_at & TRIO & Triple functional domain & 12.276 \\
\hline 210362_x_at & $P M L$ & Promyelocytic leukemia & 9.881 \\
\hline 202952_s_at & $A D A M 12$ & ADAM metallopeptidase domain 12 & 9.303 \\
\hline 205483_s_at & ISG15 & ISG15 ubiquitin-like modifier & 8.929 \\
\hline 216915_s_at & PTPN12 & Protein tyrosine phosphatase, non-receptor type 12 & 8.853 \\
\hline 200059_s_at & RHOA & Ras homolog gene family, member A & 6.685 \\
\hline 221284_s_at & $S R C$ & V-src sarcoma & 6.564 \\
\hline $\begin{array}{l}\text { AFFX-HUMISGF3A } \\
\text { M97935_MA_at }\end{array}$ & STAT1 & Signal transducer and activator of transcription 1 & 5.557 \\
\hline
\end{tabular}

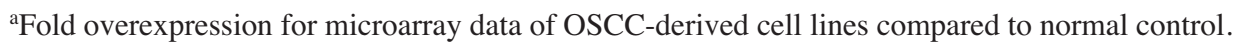


A

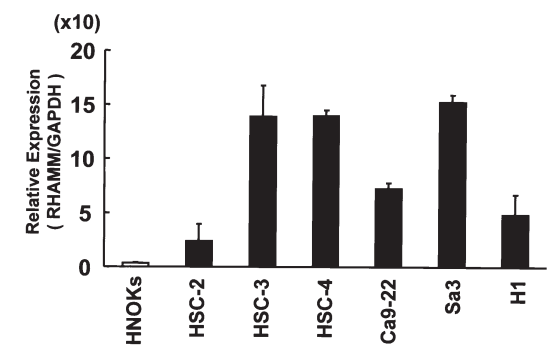

B

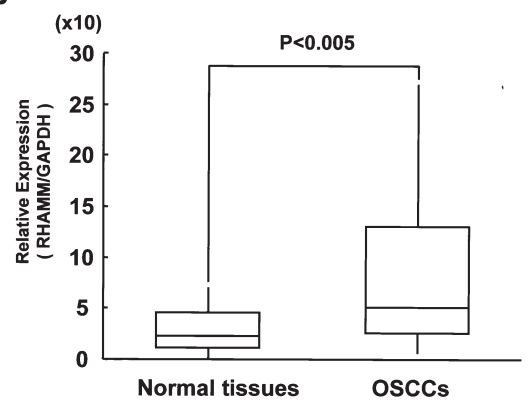

Figure 2. RHAMM mRNA expression status in OSCC-derived cell lines and primary OSCCs. (A) Quantification of mRNA levels in OSCC-derived cell lines by qRT-PCR analysis. Significant up-regulation of RHAMM mRNA expression is seen in all cell lines examined compared to mRNA expression in the HNOKs. Data are expressed as the means \pm SD. (B) Comparison of RHAMM mRNA expression levels between primary OSCCs and matched normal tissues. The relative mRNA expression levels in the normal tissue $(\mathrm{n}=50)$ and primary OSCCs $(\mathrm{n}=50)$ range from 0.08 to 7.26 (median, 2.32) and 0.53 to 27.2 (median, 5.11), respectively. A significant higher RHAMM expression is detected in primary OSCCs than matched normal tissues $(\mathrm{P}<0.005$; Mann-Whitney U test).

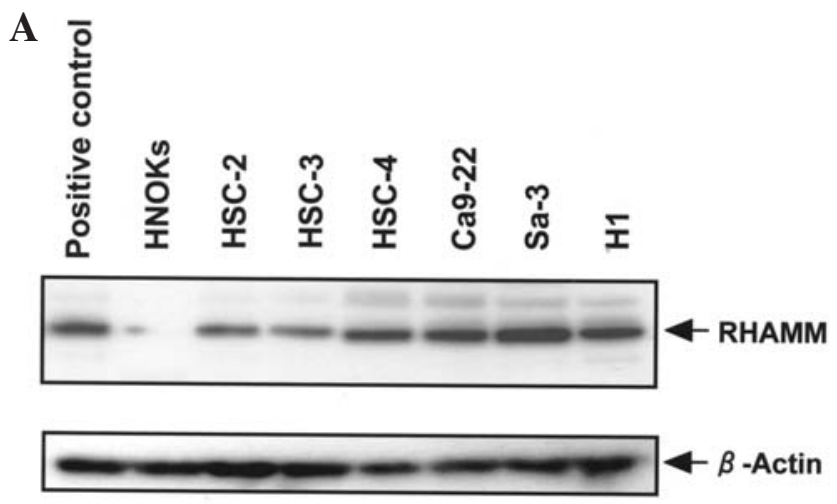

B

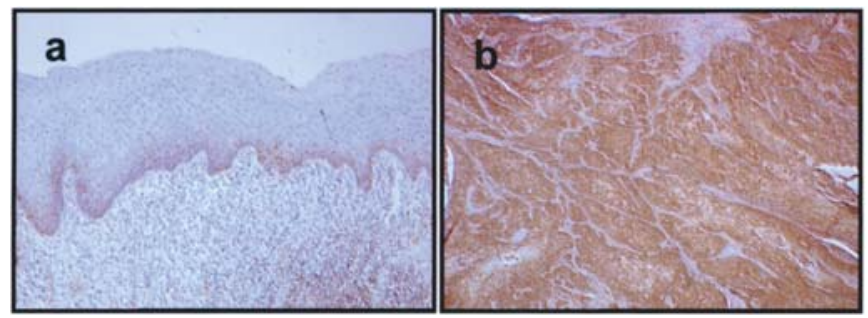

Figure 3. Representative results of RHAMM protein expression in OSCCderived cell lines, normal tissues, and primary OSCCs. (A) Western blot analysis of RHAMM protein in the OSCC-derived cell lines and the HNOKs. RHAMM protein expression is up-regulated in OSCC cell lines compared with HNOKs. (B) Immunohistochemical staining of RHAMM in normal tissue and primary OSCC. (a) Normal oral tissue exhibits negative RHAMM protein expression. (b) RHAMM-positive case of OSCC. Strong positive immunoreactivity for RHAMM is detected in the cytoplasm.
Table IV. Correlation between RHAMM expression and clinical classification in OSCCs.

Results of immunostaining No. of patients (\%)

Clinical

Total RHAMM- RHAMM+ P-value classification

Age at surgery

(years)

$\begin{array}{llll}<60 & 22 & 8(36) & 14(64) \\ \geq 60,<70 & 16 & 6(38) & 10(63) \\ \geq 70 & 14 & 4(29) & 10(71)\end{array}$

0.7004

Gender

$\begin{array}{lrrr}\text { Male } & 33 & 11(33) & 22(67) \\ \text { Female } & 19 & 7(37) & 12(63)\end{array}$

0.7978

T-primary

tumor

$\begin{array}{lrrr}\text { T1 } & 4 & 3(75) & 1(25) \\ \text { T2 } & 22 & 9(41) & 13(59) \\ \text { T3 } & 14 & 4(29) & 10(71) \\ \text { T4 } & 12 & 2(17) & 10(83) \\ \text { T1+T2 } & 26 & 12(46) & 14(54) \\ \text { T3+T4 } & 26 & 6(23) & 20(77)\end{array}$

0.9762

$\mathrm{N}$-regional

lymph node

N-

34

$14(41)$

20 (59)

0.2645

$\mathrm{N}+$

18

4 (22)

14 (78)

Stage

I

II

III

IV

$2(50)$

7 (88)

1 (12)

$<0.005$

I+II

7 (64)

24 (83)

3 (25)

$<0.001$

III+IV

9 (75)

$31(78)$

Histopathologic

type

$\begin{array}{lrrr}\text { Well } & 27 & 10(37) & 17(63) \\ \text { Moderately } & 23 & 8(35) & 15(65) \\ \text { Poorly } & 2 & 0(0) & 2(100)\end{array}$

0.6306

Tumor site

\begin{tabular}{lrrrr} 
Gingiva & 17 & $4(24)$ & $13(76)$ & \\
Tongue & 22 & $7(32)$ & $15(68)$ & 0.8345 \\
Buccal mucosa & 3 & $1(34)$ & $2(66)$ & \\
Oral floor & 6 & $3(50)$ & $3(50)$ & \\
Oropharynx & 4 & $3(75)$ & $1(25)$ & \\
\hline
\end{tabular}

${ }^{\mathrm{a}} \mathrm{P}<0.05$ statistically significant. 
A

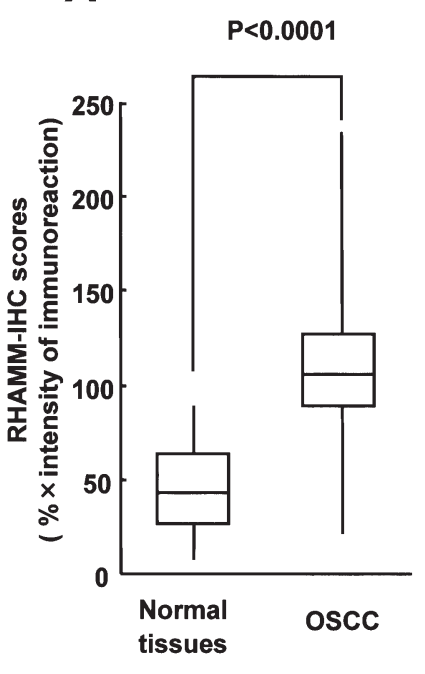

B

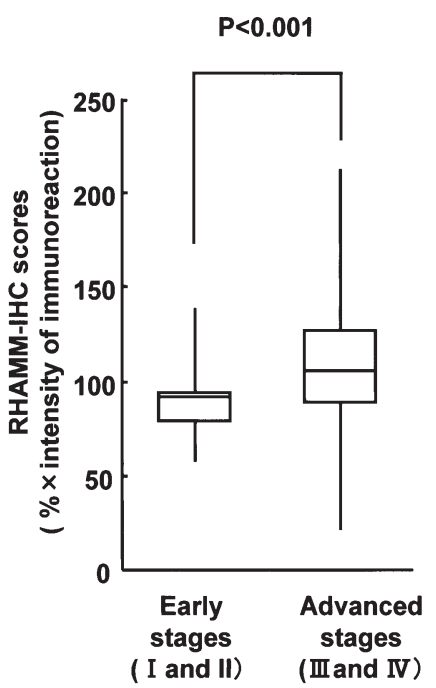

C

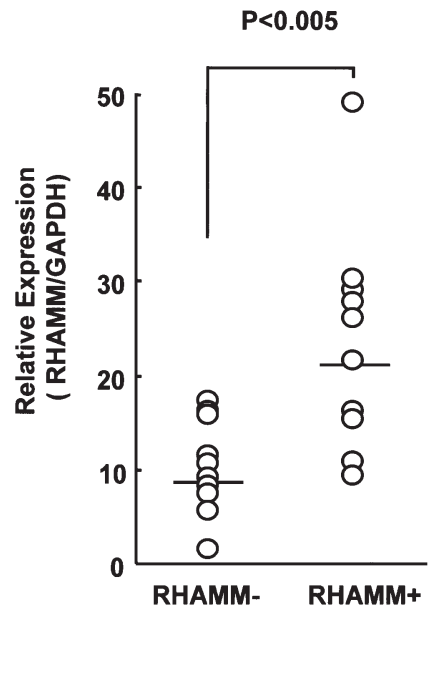

Figure 4. (A) Status of RHAMM protein expression in normal oral tissues ( $\mathrm{n}=52)$, and primary OSCCs ( $=52$ ). The RHAMM-immunohistochemistry scores are calculated as follows: RHAMM-immunohistochemistry score $=$ the mean percentage of positive tumor cells $\mathrm{x}$ staining intensity. The RHAMM immunohistochemistry scores for normal tissues and OSCCs range from 7.23 to 89.12 (median, 45.31) and 21.82 to 231.1 (median, 103.6), respectively $(\mathrm{P}<0.0001$; Mann-Whitney U test). (B) Association between RHAMM protein expression and TNM classification. The RHAMM immunohistochemistry scores for early stages (I and II) OSCC ( $\mathrm{n}=12$ ) and advanced stages (III and IV) OSCC ( $=40)$ range from 57.2 to 138.6 (median, 87.2) and 21.7 to 213.3 (median, 112.5), respectively $(\mathrm{P}<0.005$; Mann-Whitney $U$ test). (C) Comparison of RHAMM mRNA expression levels between RHAMM-positive ( $\mathrm{n}=10)$ and RHAMM-negative $(n=10)$ cases classified by immunohistochemistry analysis. Relative mRNA expression levels in negative and positive cases range from 1.3 to 17.4 (median, 8.8) and 9.8 to 48.2 (median, 20.9), respectively (P<0.005; Mann-Whitney U test). IHC, immunohistochemistry.

RHAMM expression status is summarized in Table IV. All normal oral mucosa specimens had no or significant downregulation of RHAMM expression and were considered RHAMM-negative (Fisher's exact test). Among the tumors examined, 34 of 52 cases $(65 \%)$ had RHAMM-immunoreaction in the cytoplasm of the tumor cells (Table IV). Moreover, expression of these proteins was correlated with the TNM stage grading $(\mathrm{P}<0.005)$ (Table IV) and between early stage (stages I and II) and advanced stages (III and IV) of OSCCs $(\mathrm{P}<0.001)$ (Table IV). Fig. 3B shows representative results for RHAMM protein expression in normal oral tissue and primary OSCC. The RHAMM-immunohistochemistry scores for normal tissues and OSCCs ranged from 7.23 to 89.12 (median, 45.31) and 21.82 to 231.1 (median, 103.6), respectively. The RHAMM expression levels in primary OSCCs were significantly higher than those in normal oral tissues (Mann-Whitney U test, $\mathrm{P}<0.0001$ ) (Fig. 4A). Furthermore, the RHAMM-immunohistochemistry scores for the early-stage (stages I and II) and advanced stages (III and IV) ranged from 57.2 to 138.6 (median, 87.2) and 21.7 to 213.3 (median, 112.5), respectively. The RHAMM expression levels were significantly higher in the OSCC group with advanced-stage disease compared with the group with earlystage disease (Mann-Whitney's U test, $\mathrm{P}<0.001$ ) (Fig. 4B). qRT-PCR analysis data were matched to protein expression levels studied by immunohistochemistry. RHAMM mRNA expression was significantly increased in primary tumors of randomly selected RHAMM-positive cases $(n=10)$ compared to selected RHAMM-negative cases ( $n=10$, Mann-Whitney $\mathrm{U}$ test, $\mathrm{P}<0.005$ ) (Fig. 4C). mRNA expression levels were normalized to GAPDH. Relative mRNA expression levels in negative and positive cases ranged from 1.3 to 17.4 (median, 8.8) and from 9.8 to 48.2 (median, 20.9), respectively.

\section{Discussion}

OSCC is the most frequently occurring malignancy in the oral cavity. Despite therapeutic and diagnostic advances, the 5 -year survival rate for OSCC remains approximately $50 \%$ $(18,19)$. It would therefore be helpful to discover reliable biologic marker to manage patients with OSCC; however, the constellation of genes that are instrumental in the development and progression of OSCC is unknown. Recent studies have determined gene expression profiling using microarray technology to cancer-related molecules or regions of differentially expressed genes in OSCC (9-11). However, those studies did not perform network analysis. In this context, network analysis can detect regulatory networks of differentially expressed genes and the corresponding canonical pathways. Thus, the current study was designed to identify the gene expression profiles and pathways of OSCC by means of a combination of microarray and network analysis.

Microarray analysis revealed that 166 genes were upregulated in human OSCC cells relative to HNOKs. Pathway analysis characterized seven networks from the 166 genes (Table I). In addition, gene ontology analysis identified one network (Fig. 1) that included 13 cancer-related genes that had the highest $\mathrm{P}$-values $(\mathrm{P}=2.36 \mathrm{E}-06$ to $1.23 \mathrm{E}-02)$ and top biofunctions of disease and disorders.

The development of human neoplasia is accompanied by changes in the extracellular matrix (ECM) that are essential for cell migration (20). The glycosaminoglycan hyaluronic acid (HA) is a ubiquitous component of the ECM. Increased HA expression is correlated with tumor growth and metastasis (21). Preliminary in vitro studies have suggested potential roles for two molecularly distinct cell-surface receptors 
for HA, i.e., RHAMM and CD44 play pivotal roles in tumor invasion and metastasis $(21,22)$. RHAMM was isolated originally from culture supernatants of ras-transformed murine 3T3 fibroblasts (23). In addition to its extracellular localization, RHAMM also has been shown to be an intracellular protein associated with extracellular-regulated kinase (ERK) and it plays an important role in transforming the activity of H-ras in fibroblasts (24). Increased RHAMM expression also has been reported in different invasive human tumors, such as bladder cancer (25), endometrial cancer (26), hepatocellular cancer $(27)$, breast cancer $(28,29)$ and pancreatic cancer (22).

We hypothesized that RHAMM also has potential as an emerging therapeutic target of interest for the treatment of oral cancer. However, the status of RHAMM in OSCC remains unclear. Therefore, we selected RHAMM for further investigation among the 13 cancer-related genes. To clarify the relative status of RHAMM in OSCC, we investigated the mRNA/protein expression in a series of OSCC-derived cell lines and human primary OSCCs using qRT-PCR, Western blot analysis and immunohistochemistry. Significant increases in RHAMM mRNA and protein expression were observed in the OSCC-derived cell lines examined compared with the HNOKs. We also detected a comparatively strong tumor cell-localized cytoplasmic RHAMM-immunoreaction in primary OSCCs. By evaluating the RHAMM immunohistochemistry scores using the Mann-Whitney U test, significant RHAMM up-regulation was evident in the primary OSCCs ( $\mathrm{P}<0.0001$, OSCC vs. corresponding normal tissues) compared with normal tissues. qRT-PCR analysis data were matched to protein expression levels studied by Western blot analysis and immunohistochemistry. The RHAMM protein expression levels in primary OSCCs were significantly associated with TNM stage grading $(\mathrm{P}<0.005)$. Moreover, the state of the protein RHAMM expression differed significantly between the early stages (I and II) and the advanced stages (III and IV) (Mann-Whitney U test, $\mathrm{P}<0.001)$. Our results suggested a significant association between RHAMM and the clinical stages of tumors, with up-regulation of RHAMM in the advanced stages (III and IV) of OSCC, which may result in tumor aggressiveness and progression of OSCC. A recent study reported that RHAMM overexpression enhanced sensitivity of aurora kinase inhibitor (VX-680) to multiple myeloma cells (30). In addition, vaccine therapy in which dendritic cells transfected with modified RHAMM mRNA were used induced strong antitumor effects in vitro (31). From the therapeutic standpoint, this evidence may provide a novel/effective approach to treat human OSCCs.

In summary, we found novel specific networks of the cancer-related genes in OSCC cells and identified several candidate genes for molecular targeting, especially for RHAMM. Moreover, our findings contribute to the understanding of key biologic functions and pathways of certain up-regulated genes associated with OSCC, and the expression status of RHAMM may play an important role in the oral carcinogenesis.

\section{Acknowledgments}

We thank Lynda C. Charters for editing this report.

\section{References}

1. Landis SH, Murray T, Bolden S and Wingo PA: Cancer statistics. CA Cancer J Clin 49: 8-31, 1999.

2. Funk GF, Karnell LH, Robinson RA, Zhen WK, Trask DK and Hoffman HT: Presentation, treatment and outcome of oral cavity cancer: a national cancer data base report. Head Neck 24: 165-180, 2002.

3. Lippman SM, Sudbo J and Hong WK: Oral cancer prevention and the evolution of molecular-targeted drug development. J Clin Oncol 23: 346-356, 2005.

4. Vokes EE, Weichselbaum RR, Lippman SM and Hong WK: Head and neck cancer. N Engl J Med 328: 184-194, 1993.

5. Macfarlane GJ, Zheng T, Marshall JR, et al: Alcohol, tobacco, diet and the risk of oral cancer: a pooled analysis of three case-control studies. Eur J Cancer B Oral Oncol 31: 181-187, 1995.

6. Mashberg A, Boffetta P, Winkelman R and Garfinkel L: Tobacco smoking, alcohol drinking, and cancer of the oral cavity and oropharynx among US veterans. Cancer 72: 1369-1375, 1993.

7. Fearon ER and Vogelstein B: A genetic model for colorectal tumorigenesis. Cell 61: 759-767, 1990.

8. Marshall CJ: Tumor suppressor genes. Cell 64: 313-326, 1991.

9. Odani T, Ito D, Li MH, et al: Gene expression profiles of oral leukoplakia and carcinoma: genome-wide comparison analysis using oligonucleotide microarray technology. Int J Oncol 28: 619-624, 2006.

10. Tomioka H, Morita K, Hasegawa S and Omura K: Gene expression analysis by cDNA microarray in oral squamous cell carcinoma. J Oral Pathol Med 35: 206-211, 2006.

11. Leethanakul C, Knezevic V, Patel V, et al: Gene discovery in oral squamous cell carcinoma through the head and neck cancer genome anatomy project: confirmation by microarray analysis. Oral Oncol 39: 248-258, 2003.

12. Koike H, Uzawa K, Nakashima D, et al: Identification of differentially expressed proteins in oral squamous cell carcinoma using a global proteomic approach. Int J Oncol 27: 59-67, 2005 .

13. Endo Y, Uzawa K, Mochida Y, et al: Sarcoendoplasmic reticulum $\mathrm{Ca}(2+)$ ATPase type 2 downregulated in human oral squamous cell carcinoma. Int J Cancer 110: 225-231, 2004.

14. Kasamatsu A, Uzawa K, Nakashima D, et al: Galectin-9 as a regulator of cellular adhesion in human oral squamous cell carcinoma cell lines. Int J Mol Med 16: 269-273, 2005.

15. Shimada K, Uzawa K, Kato M, et al: Aberrant expression of RAB1A in human tongue cancer. Br J Cancer 92: 1915-1921, 2005.

16. Tanaka C, Uzawa K, Shibahara T, Yokoe H, Noma H and Tanzawa $\mathrm{H}$ : Expression of an inhibitor of apoptosis, survivin, in oral carcinogenesis. J Dent Res 82: 607-611, 2003.

17. Assmann V, Marshall JF, Fieber C, Hofmann M and Hart IR: The human hyaluronan receptor RHAMM is expressed as an intracellular protein in breast cancer cells. J Cell Sci 111: 1685-1694, 1998

18. Okamoto M, Nishimine M, Kishi M, et al: Prediction of delayed neck metastasis in patients with stage I/II squamous cell carcinoma of the tongue. J Oral Pathol Med 31: 227-233, 2002.

19. Weinberg MA and Estefan DJ: Assessing oral malignancies. Am Fam Physician 65: 1379-1384, 2002.

20. Itano N, Sawai T, Miyaishi O and Kimata K: Relationship between hyaluronan production and metastatic potential of mouse mammary carcinoma cells. Cancer Res 59: 2499-2504, 1999.

21. Delpech B, Girard N, Bertrand P, Courel MN, Chauzy C and Delpech A: Hyaluronan: fundamental principles and applications in cancer. J Intern Med 242: 41-48, 1997.

22. Abetamann V, Kern HF and Elsasser HP: Differential expression of the hyaluronan receptors CD44 and RHAMM in human pancreatic cancer cells. Clin Cancer Res 2: 1607-1618, 1996.

23. Turley EA, Moore D and Hayden LJ: Characterization of hyaluronate binding proteins isolated from 3T3 and murine sarcoma virus transformed $3 \mathrm{~T} 3$ cells. Biochemistry 26 : 2997-3005, 1987.

24. Zhang S, Chang MC, Zylka D, Turley S, Harrison R and Turley EA: The hyaluronan receptor RHAMM regulates extracellular-regulated kinase. J Biol Chem 273: 11342-11348, 1998. 
25. Kong QY, Liu J, Chen XY, Wang XW, Sun Y and Li H: Differential expression patterns of hyaluronan receptors CD44 and RHAMM in transitional cell carcinomas of urinary bladder. Oncol Rep 10: 51-55, 2003.

26. Rein DT, Roehrig K, Schondorf T, et al: Expression of the hyaluronan receptor RHAMM in endometrial carcinomas suggests a role in tumour progression and metastasis. J Cancer Res Clin Oncol 129: 161-164, 2003.

27. Yang CW, Su JY, Tsou AP, et al: Integrative genomics based identification of potential human hepatocarcinogenesis-associated cell cycle regulators: RHAMM as an example. Biochem Biophys Res Commun 330: 489-497, 2005.

28. Assmann V, Gillett CE, Poulsom R, Ryder K, Hart IR and Hanby AM: The pattern of expression of the microtubulebinding protein RHAMM/IHABP in mammary carcinoma suggests a role in the invasive behaviour of tumour cells. J Pathol 195: 191-196, 2001.
29. Wang C, Thor AD, Moore DH, et al: The overexpression of RHAMM, a hyaluronan-binding protein that regulates ras signaling, correlates with overexpression of mitogen-activated protein kinase and is a significant parameter in breast cancer progression. Clin Cancer Res 4: 567-576, 1998

30. Shi Y, Reiman T, Li W, et al: Targeting aurora kinases as therapy in multiple myeloma. Blood 109: 3915-3921, 2007.

31. Amano T, Kajiwara K, Yoshikawa K, et al: Antitumor effects of vaccination with dendritic cells transfected with modified receptor for hyaluronan-mediated motility mRNA in a mouse glioma model. J Neurosurg 106: 638-645, 2007. 